\title{
Erratum: Action-Specific Disruption of Perceptual Confidence
}

Psychological Science 2015, Vol. 26(4) 551 (C) The Author(s) 2015 Reprints and permissions: sagepub.com/journalsPermissions.nav DOI: $10.1177 / 0956797615578854$ pss.sagepub.com

@SAGE

Fleming, S. M., Maniscalco, B., Ko, Y., Amendi, N., Ro, T., \& Lau, H. (2015). Action-specific disruption of perceptual confidence. Psychological Science, 26, 89-98. (Original DOI: 10.1177/0956797614557697)

In Figure 3 of this article, the labels in the key were accidentally switched. The key should have indicated that the red symbols show results for incongruent trials and the blue symbols show results for congruent trials. The corrected figure is printed below.

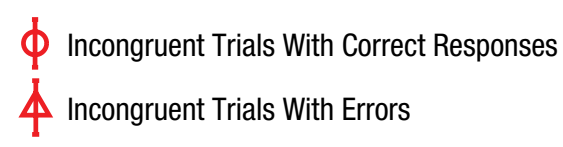

a

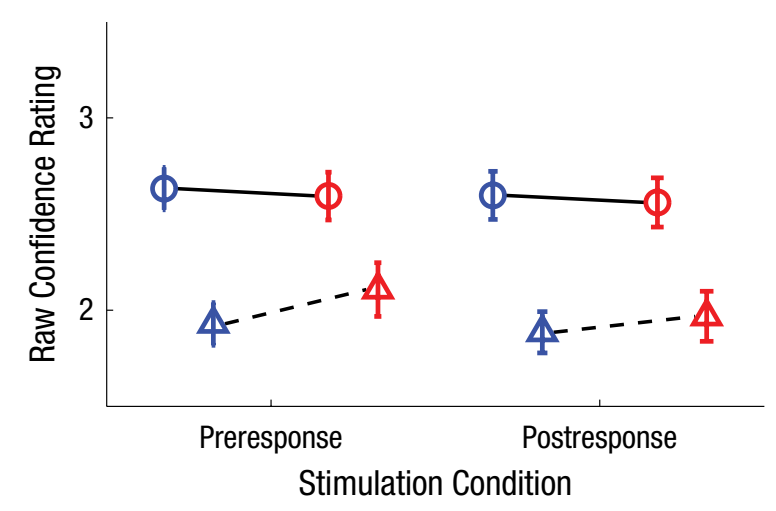

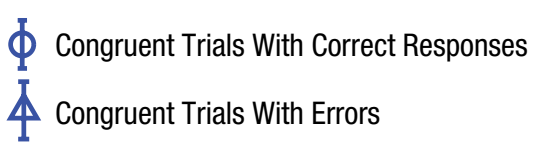

b

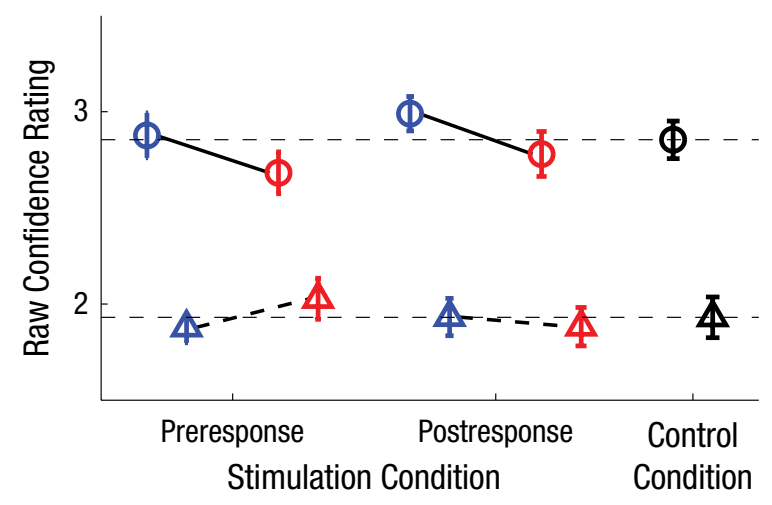

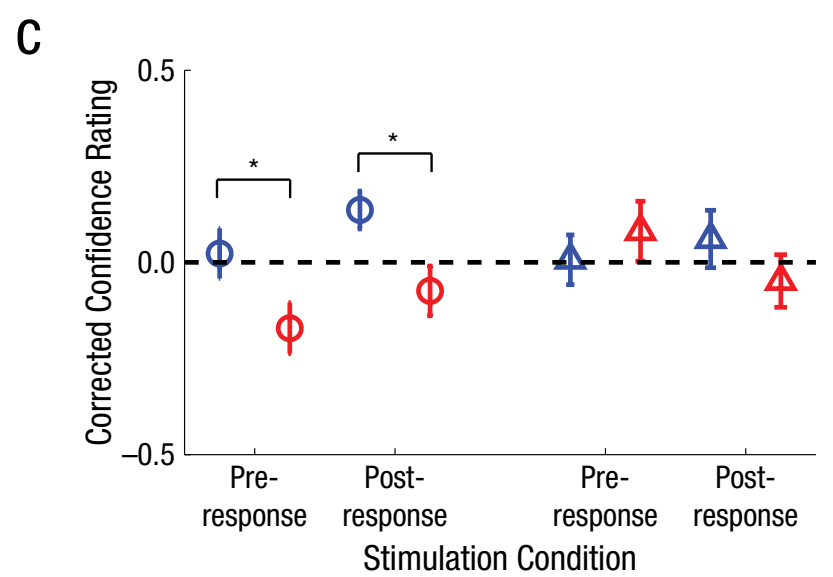

Fig. 3. Confidence ratings in the group that received transcranial magnetic stimulation (TMS) in the dorsal premotor cortex in (a) Experiment 1 and (b, c) Experiment 2. The graphs in (a) and (b) show raw mean confidence ratings as a function of stimulation condition, response accuracy, and congruence. In (b), results are also shown for a control condition (in which TMS was not applied) for trials responded to correctly and incorrectly. Dashed lines reflect mean confidence in the control condition. The graph in (c) shows baseline-corrected confidence data in Experiment 2 as a function of response accuracy. Baseline-corrected confidence data were calculated by subtracting mean raw confidence ratings on no-TMS trials from mean raw confidence ratings on TMS trials. Error bars reflect standard errors of the mean. Asterisks indicate a significant difference between TMS conditions $(p<.05)$. 